Revista de Investigación Educativa 27

julio-diciembre, 2018 | ISSN 1870-5308 | Xalapa, Veracruz

Instituto de Investigaciones en Educación | Universidad Veracruzana

\title{
Uandakurhintskua - biografías lingüísticas de docentes p'urhepechas \\ De la escolaridad traumática a la creación de un modelo educativo alternativo
}

\section{Uandakurhintskua - P'urhepecha teachers and their linguistic biographies \\ From trauma at school to the making of an alternative model of education}

\author{
Rainer Enrique Hamel ${ }^{\mathrm{a}}$ \\ Ana Carolina Hecht ${ }^{b}$ \\ Ana Elena Erape Baltazar \\ Helmith Betzabé Márquez Escamillad
}

Recibido: 25 de enero de 2018

Aceptado: 24 de abril de 2018

En sus entrevistas autobiográficas, analizadas con la metodología de la "entrevista autobiográfica narrativa” (Fritz Schütze), las maestras y maestros p'urhepechas del proyecto escolar “T’arhexperakua-Creciendo Juntos" de Michoacán, México, construyen en retrospectiva un contraste entre su propia experiencia escolar, traumática y profundamente insatisfactoria, y su práctica profesional de equipo, donde intentan ofrecerle una alternativa más pertinente y feliz a sus alumnos. Los docentes narran su propia experiencia de primaria indígena, donde no lograron aprender bien la "otra" lengua ni los contenidos escolares previstos. Recién en su formación como maestros se tornaron bilingües y adquirieron un orgullo por su identidad étnica. Movilizando el enorme potencial de la cultura y lengua propias, construyeron un currículo en educación intercultural bilingüe en más de veinte años de experiencia colectiva. El estudio aporta una introspección valiosa en la motivación y conciencia etnolingüística profesional de un equipo de docentes que comparten un proyecto escolar reconocido nacional e internacionalmente.

Palabras clave: Educación indígena; autobiografías; bilingüismo.

\footnotetext{
a Universidad Autónoma Metropolitana, Departamento de Antropología, Ciudad de México, México. $₫$ hamel@xanum.uam.mx

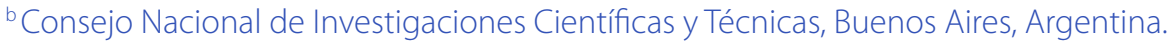

‘Universidad Autónoma Metropolitana, Departamento de Antropología, Ciudad de México, México.

dUniversidad Autónoma Metropolitana, Departamento de Antropología, Ciudad de México, México.
} 
Rainer Enrique Hamel, Ana Carolina Hecht, Ana Elena Erape Baltazar, Helmith Betzabé Márquez Escamilla

In their narrative autobiographical interviews (Fritz Schütze), the P'urhepecha teachers of the "T'arhexperakua-Growing together" school project in central Mexico construe in retrospect a clear contrast between their own traumatic school experience as pupils based on "Spanish only" instruction, and their professional practice today. Then they did not get an opportunity of learning the "other language" nor the relevant content matters. It was only during their teacher training that they became truly bilingual and developed an ethnic consciousness. Now, they want to offer a better alternative to their indigenous pupils. Mobilizing the enormous potential of their own culture and P'urhepecha as a language of identity and instruction, they have developed a truly intercultural bilingual programme based on mother tongue education over a timespan of twenty years. The study offers a valuable insight into the motivation and the professional ethnolinguistic consciousness of a team whose school project is well respected in Mexico and abroad.

Keywords: Indigenous education; autobiographies; bilingualism.

\title{
Uandakurhintskua - biografías lingüísticas de docentes p'urhepechas
}

De la escolaridad traumática a la creación de un modelo educativo alternativo

\author{
Uandakurhintskua - P'urhepecha teachers \\ and their linguistic biographies
}

From trauma at school to the making of an alternative model of education

\section{El proyecto escolar "T'arhexperakua-Creciendo Juntos"}

¿Q ué nos pueden revelar los relatos autobiográficos de maestras y maestros indígenas sobre su socialización lingüística, sus trayectorias escolares y sus orientaciones pedagógicas para entender mejor su desempeño como docentes en un proyecto escolar destacado, intercultural-bilingüe y de gestación propia?

El proyecto escolar "T'arhexperakua-Creciendo Juntos" ha funcionado sin interrupción desde 1995 en las escuelas primarias p'urhepechas de San Isidro y Uringuitiro en 
Michoacán, y es bastante conocido en México y más allá de sus fronteras (Dietz, 2012; López, 2009b). En un inicio los docentes tomaron una decisión muy importante. El programa típico de "castellanización", es decir, el intento de enseñar los contenidos curriculares en español, había fracasado estrepitosamente, al igual que en las demás escuelas de la comarca. Esto ocurría así porque sus alumnos ingresaban en su mayoría como monolingües en lengua indígena a la escuela, debido a la alta vitalidad etnolingüística en la región.

Frente a esta realidad, los maestros y maestras resolvieron de manera audaz, y a la vez correcta, operar un cambio e impartir todas las materias en p'urhepecha, ya que en su lengua materna los alumnos comprendían mucho mejor lo que aprendían; se producía además una participación más activa y con un mayor grado de satisfacción (Alonso, Alonso, Cano \& Cortés, 2004). Colocaron en el centro de su quehacer la lengua indígena como principal herramienta de alfabetización y enseñanza-aprendizaje de todas las asignaturas. Para ellos, la lengua propia constituye el núcleo de la identidad y de la fuerza cultural que impulsa a maestros y alumnos. Es hoy en día la lengua legítima, no marcada, en todas las actividades de las escuelas, y no tiene que pedir permiso para entrar, como en tantas otras escuelas indígenas.

A pesar de su éxito y creciente reconocimiento, el proyecto tiene que enfrentarse a muchas resistencias en el sector educativo, porque rompe con las inercias típicas de la educación indígena en México: la práctica del menor esfuerzo posible, la aplicación mecánica de los programas y libros de texto diseñados para alumnos hispanohablantes, la castellanización y la exclusión o subordinación de las lenguas y culturas indígenas como expresión de la persistente discriminación.

Lo que quizás más fascina a los observadores y a la vez causa preocupación e irritación en algunos, es que el proyecto ha demostrado en la práctica un funcionamiento que muchos maestros indígenas, las mismas autoridades educativas y sectores importantes de la sociedad mexicana creen que es imposible: alfabetizar y aprender con éxito el conjunto de contenidos y competencias de la cultura propia y del currículo nacional en lengua indígena, y apropiarse al mismo tiempo del español como segunda lengua.

En otras palabras, se fue creando un currículo bilingüe e intercultural de raíz a partir del propio esfuerzo que se llevó a la práctica todos los días, y se les proveyó a los alumnos y alumnas p'urhepechas una educación mucho más pertinente, exitosa y feliz que la educación que se imparte en muchas otras escuelas indígenas. De este modo, las reivindicaciones etno-políticas del reconocimiento de las lenguas y culturas indígenas se integran de manera pertinente con un modelo pedagógico muy superior a las malas tradiciones escolares, como se explica en detalle en las investigaciones existentes sobre 
el proyecto colaborativo escolar (Hamel, 2010, 2016). Aquí, la interculturalidad y el bilingüismo dejan de ser términos decorativos que se suman a la palabra educación, que en muchos casos simulan cambios en las escuelas, pero reproducen en la cotidianeidad las mismas prácticas, representaciones y discursos de la educación castellanizadora y monocultural. Por el contrario, los docentes rediseñaron los contenidos desde esta propuesta, tomando en cuenta las prácticas comunicativas bilingües y la situación cotidiana de interculturalidad, es decir, la acción día a día de los profesores que desarrollan un conocimiento propio en el aula a partir de su cultura para poder dialogar con otros conocimientos, como un modelo de enriquecimiento del ser y del saber.

Desde 1999 el proyecto escolar cuenta con el acompañamiento de un equipo de pedagogos, sociolingüistas y antropólogos de la Universidad Autónoma Metropolitana (UAM), la Universidad Pedagógica Nacional (UPN) y de otras instituciones, quienes se organizaron en el programa permanente "Comunidad Indígena y Educación Intercultural Bilingüe” (CIEIB). ${ }^{1}$ En una labor colaborativa de larga duración se fueron fusionando los dos equipos, y hoy los y las investigadoras externas forman parte también del proyecto escolar T’arhexperakua y viceversa. Desarrollamos conjuntamente varias líneas de investigación y acción ${ }^{2}$ en cuyo centro se ubica la elaboración, paso a paso, y la inmediata implementación de los avances de un currículo experimental propio, que enriquece y transforma el currículo oficial de primaria.

\section{Análisis de las trayectorias escolares}

\subsection{La entrevista autobiográfica narrativa: primer acercamiento}

En algún momento del trabajo colaborativo que se centraba en los aprendizajes, las prácticas escolares y la elaboración curricular, surgió la preocupación por cono-

1. Tiene su sede en el Departamento de Antropología de la Universidad Autónoma Metropolitana, Unidad Iztapalapa, bajo la dirección de Rainer Enrique Hamel. Agradecemos el financiamiento de nuestro trabajo por varios proyectos consecutivos de CONACYT y de la Fundación Ford.

2. Las áreas del trabajo colaborativo son las siguientes: 1) Investigación básica y aplicada: Procesos de adquisición y desarrollo de L1 y L 2 en sus cuatro habilidades en el aula; evaluación de estos procesos a través de la evaluación mediante pruebas; identificación de fortalezas y problemas en el proceso de enseñanza-aprendizaje. 2) Desarrollo curricular. 3) Formación de profesores en servicio desde el aula. 4) Formación de posgrado (maestría y doctorado) de miembros del equipo. 5) Producción de materiales y documentales en video. 
Rainer Enrique Hamel, Ana Carolina Hecht, Ana Elena Erape Baltazar, Helmith Betzabé Márquez Escamilla

cer de manera más sistemática las biografías educativas de los maestros y maestras p’urhepechas; pensábamos que tal conocimiento más explícito pudiera llevarnos a una mayor comprensión de las intervenciones educativas de los docentes en el aula y sus orientaciones respectivas. ¿Cuál ha sido el componente individual y biográfico que les permitió a maestros y maestras impulsar este proyecto escolar? ¿Qué adversidades debieron enfrentar en su camino de ser alumnos y estudiantes primero, para transformarse en maestros después? ¿Qué les ha dado fuerza, insumos y orientación para seguir adelante? ¿Qué elementos de satisfacción personal y profesional les ha brindado su proyecto? Por otro lado, ¿cuáles han sido también las dudas, incertidumbres y resquemores que surgen, inevitablemente, en su quehacer cotidiano como docentes? ¿De qué manera sus saberes, creencias e ideologías se han transformado en el transcurso de una extensa trayectoria docente que se ha convertido sin duda en un proyecto de vida para muchos de ellos? Y, por último, ¿qué elementos nos pueden aportar sus biografías escolares - o sus vidas en el transcurrir por la escuela y sus devenires como sujetos bilingües - para considerarlos, incluso extrapolarlos, al diseño actual de la educación intercultural bilingüe?

Para responder a algunas de estas interrogantes hemos iniciado una investigación conjunta. ${ }^{3}$ Presentamos aquí una primera indagación que se propone reconstruir el papel de las trayectorias educativas y lingüísticas en las autobiografías escolares de maestras y maestros y sus referencias al proyecto escolar T'arhexperakua. Para este fin, realizamos unas entrevistas autobiográficas narrativas $(\text { EAN })^{4}$ con algunos docentes de las escuelas de San Isidro y Uringuitiros.

La metodología subyacente a estas entrevistas propone solicitarle al entrevistado una narración detallada de su vida, en este caso, de su historia escolar, desde su ingre-

3. La investigación fue propuesta y encabezada por Ana Carolina Hecht, investigadora del CONICET y visitante de posdoctorado proveniente de Argentina, quien participó por varios meses en nuestro proyecto en 2011. Ver la investigación paralela con los Toba en Argentina (Hecht, 2015). Agradecemos el otorgamiento de una beca Unesco de la Fundación Keizo Obuchidel Japón que hizo posible su estancia en México.

4. El concepto preciso es de Fritz Schütze. Nos basamos en el desarrollo teórico-metodológico de este grupo de sociólogos alemanes que retomamos y ampliamos en México (Appel, 2005; Guerra, 2009; Kohler, 1993; Muñoz, 2006; Riemann \& Schütze, 1991; Schütze, 1983, 1992a, 1992b; Sierra, 1992). Para enfoques y usos diferentes en México, consúltese Serrano Castañeda y Ramos Morales (2014) y de Souza Serrano y Ramos (2014).

5. Las entrevistas fueron realizadas por Carolina Hecht, quien, como investigadora externa, sabía menos del proyecto y de la vida de los docentes que el resto del grupo. Esta consideración metodológica ayudó a crear una situación realista en las entrevistas, donde los docentes tenían que explicar sus trayectorias y actuaciones a una persona de afuera. 
Rainer Enrique Hamel, Ana Carolina Hecht, Ana Elena Erape Baltazar, Helmith Betzabé Márquez Escamilla

so a las instituciones educativas como alumno hasta su actual desempeño como maestro, sin que la entrevistadora interfiriera con preguntas o comentarios que podrían haber alterado el hilo conductor y las categorizaciones del relato. El procedimiento permite aproximarse a la aprehensión cognitiva de las estructuras del mundo de los sujetos a través de sus textos narrativos y descriptivos. ${ }^{6}$ Las entrevistas se iniciaron con la siguiente pregunta disparadora: " ¿Cómo fue su experiencia cuando entró a la escuela como alumno? ¿Qué fue lo que más le llamó la atención de la escuela?”7

En este acercamiento a las autobiografías narrativas no podemos abarcar todos los temas relevantes en el contexto de una colaboración escuela-universidad de larga duración que ha producido gran cantidad de materiales y también de interrogantes. Dejaremos para estudios venideros una exposición exhaustiva del análisis formal de las entrevistas. Postergamos también el interesante reto de relacionar los contenidos y las construcciones ideológicas de las narrativas con otras fuentes de información, como son las extensas observaciones de los desempeños pedagógicos de maestras y maestros. Nos queda por explorar, por tanto, hasta qué punto, en este caso, los docentes logran construir una congruencia entre sus constructos explicativos y justificativos y sus actuaciones; o si, por el contrario, se producen las sistemáticas discrepancias entre las prácticas sociales y sus representaciones, que hemos encontrado en otros estudios similares (Hamel \& Muñoz Cruz, 1988; Lafont, 1979; ver también el apartado 2).

En lo que sigue llevaremos a cabo un primer análisis de contenidos de los textos autobiográficos; sistematizaremos las categorías que surgen en ellos para interpretarlas a la luz de nuestro conocimiento e involucramiento con el quehacer cotidiano y la construcción colaborativa de una práctica social pedagógica en las escuelas participantes.

6. Se basa en la hipótesis teórica de que la narración de experiencias propias, relatadas ad hoc y en primera persona, tiende a constituirse en un evento robusto capaz de construir un esquema discursivo tan sólido que éste se aproxima a la acción reportada misma, y reconstruye en buena medida las orientaciones de la realidad de acción de los sujetos (Riemann \& Schütze, 1991).

7. Cabe especificar que la lengua en la que se realizaron las entrevistas fue el español, la lengua académica "más fuerte" de todos los docentes. En una etapa futura se prevé replicar o ampliar estas entrevistas en lengua p'urhepecha. Una vez transcritas las entrevistas, éstas fueron entregadas a las y los maestros participantes. Como el equipo sigue trabajando con las escuelas, los avances de estudios en curso se presentan periódicamente en los talleres para crear un debate, pero también para ser incluidos directamente en la planeación educativa. En dos talleres realizados en 2015 y 2016 sobre la relevancia de los "proyectos de vida" de los docentes (particularmente en cuanto a sus orientaciones lingüísticas y pedagógicas, y cómo influyen en el desarrollo del proyecto escolar y los desempeños individuales), se incluyeron los resultados de la investigación de manera indirecta, sin identificar individualmente a los actores ante la comunidad magisterial. Como ya es tradición de muchos años en nuestro trabajo colaborativo, se programará un nuevo taller una vez que este texto esté publicado. 
Rainer Enrique Hamel, Ana Carolina Hecht, Ana Elena Erape Baltazar, Helmith Betzabé Márquez Escamilla

\section{2 Los maestros y maestras establecen sus categorías de relevancia}

Las seis entrevistas realizadas reflejan una selección representativa de la planta docente de las dos escuelas. ${ }^{8}$ La muestra elegida se compone de cuatro hombres y dos mujeres p'urhepechas bilingües que cubren una amplia franja etaria entre los 24 y los 42 años. Cuatro tienen el p'urhe como lengua materna, dos, el español; tres son de San Isidro y los restantes de otras localidades cercanas. Su experiencia profesional oscila entre uno y 18 años de servicio (Tabla 1).

\section{Tabla 1. Caracterización del perfil lingüístico y profesional de los entrevistados}

\begin{tabular}{|c|c|c|}
\hline \multirow{2}{*}{ Género } & Masculino & Femenino \\
\hline & 4 & 2 \\
\hline \multirow[t]{2}{*}{ Lenguaje } & $\begin{array}{c}\text { P'urhepecha lengua } \\
\text { materna }\end{array}$ & Español lengua materna \\
\hline & 4 & 2 \\
\hline \multirow{2}{*}{ Franja etaria } & 24-34 años & 35-42 años \\
\hline & 3 & 3 \\
\hline \multirow{2}{*}{$\begin{array}{l}\text { Experiencia } \\
\text { Docente }\end{array}$} & 1-8 años & 9-18 años \\
\hline & 2 & 4 \\
\hline \multirow{2}{*}{$\begin{array}{l}\text { Lugar de origen / } \\
\text { Residencia }\end{array}$} & San Isidro & Alrededores \\
\hline & 3 & 3 \\
\hline
\end{tabular}

El estudio de las trayectorias de las dos lenguas en las biografías escolares nos permite reconstruir las ideologías lingüísticas como sistemas de interpretación y orientación (Hamel, 2000), con componentes explícitos e implícitos, de la acción propia en los procesos de socialización. Este tipo de estudios entre hablantes bilingües es un cam-

8. En el momento del levantamiento de los datos (2011) laboraba un total de siete docentes en la escuela de Uringuitiro. Cinco eran hombres y dos, mujeres; la lengua de todos los docentes era el p'urhepecha. En la escuela de San Isidro trabajaban 18 docentes, 11 hombres y siete mujeres. Del conjunto de docentes, cuatro tenían el español como lengua materna y habían adquirido el p'urhepecha posteriormente; diez tenían el p'urhepecha como lengua materna y cuatro pasaron por una socialización primaria bilingüe relativamente equilibrada. 
Rainer Enrique Hamel, Ana Carolina Hecht, Ana Elena Erape Baltazar, Helmith Betzabé Márquez Escamilla

po aún poco explorado en el contexto de las investigaciones sobre educación intercultural bilingüe en América Latina. ${ }^{9}$

Las ideologías lingüísticas refieren tanto a aquellas ideas que sostienen los hablantes sobre el lenguaje como al impacto que esas ideas tienen en el habla y las prácticas cotidianas (Woolard, 1998). Son "un conjunto de creencias sobre el lenguaje, articulado por los usuarios como una racionalización o justificación de la estructura y el uso percibido de la lengua" (Silverstein, 1979, como se citó en Woolard, 1998, p. 1), que median entre el signo y la situación social. Las ideologías lingüísticas enlazan las prácticas comunicativas cotidianas con nociones del campo social, ya que las ideologías se respaldan en las prácticas de los sujetos que, a la vez, se sustentan en ideas sobre la vida social. Es decir, las ideas no se restringen al lenguaje, sino que remiten a su vinculación con otros aspectos de la vida cotidiana, como las nociones locales de identidad y las etapas de la vida y de enseñanza-aprendizaje.

En las primeras investigaciones sociolingüísticas sobre el tema se ha observado que, cuando existen conflictos etnolingüísticos y una asimetría diglósica entre las lenguas involucradas, se producen típicamente contradicciones sistemáticas entre prácticas discursivas y representaciones, como lo muestran los estudios occitanos en Francia, pioneros de la sociolingüística (Lafont, 1979, 1980), exploraciones en África (Manessy \& Wald, 1984), pero también las investigaciones en latitudes latinoamericanas que relacionan las prácticas discursivas con el nivel reflexivo de la conciencia lingüística (Hamel \& Muñoz, 1982; Muñoz, 1983, 2009). Una cuestión es cómo habla la gente, y otra muy distinta es cómo dice que habla. Luego se producen procesos de racionalización discursiva que intentan establecer una nueva coherencia entre componentes contradictorios en las prácticas, biografías y reflexiones.

Por esta razón, resulta interesante vincular esas racionalizaciones en el marco de los procesos de socialización lingüística con el fin de comprender los complejos escenarios educativos actuales. La sistematización de estos temas en contextos bilingües y multiculturales permite comprender los procesos de cambio y transformación que atraviesan las lenguas en contacto y conflicto, los vínculos entre sus usos dentro y fuera de la escuela, como también los procesos de identificación étnica de sus hablantes.

Iniciemos el análisis de las autobiografías recabadas. En sus discursos surgieron cuatro temas significativos en la construcción que los sujetos entrevistados elaboraron de sus biografías escolares:

9. Trabajos pioneros sobre estas expresiones metalingüísticas son los estudios de Héctor Muñoz Cruz (1983, 2006, 2009; Hamel \& Muñoz Cruz, 1982, 1988). 
1. El devenir bilingüe implicó transitar por un camino conflictivo.

2. La escuela indígena primaria no fue bilingüe y desatendió sus necesidades lingüísticas.

3. Se produjeron cambios y quiebres en la valoración de la lengua y cultura p'urhepechas.

4. Los estudios universitarios constituyen una fuente de transformación personal que permite la superación profesional permanente como clave de la vida.

A continuación, desarrollaremos cada uno de estos temas, apoyándonos en los testimonios de los mismos docentes indígenas, en nuestro análisis y nuestro conocimiento de la situación escolar.

\section{3 El devenir bilingüe implicó transitar por un camino conflictivo}

En sus relatos biográficos, todos los maestros y maestras manifestaron algún conflicto en su propio devenir como sujetos bilingües, y esta particular situación trasciende la división entre aquellos que iniciaron su vida como monolingües en p'urhepecha o en español (Tabla 1). La adquisición exitosa de la otra lengua ocurrió en una fase bastante tardía, durante el bachillerato o incluso después, en sus estudios superiores, caracterizada como un proceso difícil y atravesado por tensiones. Definitivamente no aconteció en la escuela primaria, supuestamente bilingüe, tal como veremos en el siguiente apartado. Resulta muy significativo que los docentes mismos sacaron a relucir el tema de las lenguas a través de los escollos vividos en la escuela por la carencia de competencias lingüísticas en la segunda lengua, sea ésta el español o el p'urhepecha.

Los profesores que eran monolingües en p'urhepecha aprendieron el español por necesidad y por la vergüenza que les causaba hablar p'urhepecha y saber mal el español (Tabla 2). De los monolingües hispanohablantes, algunos aprendieron el p'urhepecha ya en la escuela para relacionarse con los compañeros; pero nunca en el salón, sino en el recreo y en interacciones no controladas por los profesores, ya que la lengua indígena vivía excluida del aula (Tabla 3 ). Es un hecho conocido que se da bajo determinadas condiciones, incluso en escuelas donde la lengua indígena se encuentra en una fase avanzada de desplazamiento, que los alumnos hispanohablantes avanzan de todos modos en la adquisición de esta lengua (Hamel, 2009). 


\section{Tabla 2. Del p'urhepecha al español. Trayectoria lingüística de maestras y maestros entrevistados}

\begin{tabular}{|c|c|c|c|c|}
\hline \multicolumn{5}{|c|}{$\begin{array}{l}\text { Profesores de lengua materna p'urhepecha: } \rightarrow \text { adquisición del español L2 } \\
\text { Motivación: vergüenza y estigmatización por no hablar E, necesidad educativa. } \\
\text { Proceso: traumático y conflictivo, poco eficiente. } \\
\text { Resultado: bilingüismo coordinado tardío, muchas veces asimétrico. }\end{array}$} \\
\hline \multicolumn{5}{|c|}{ Trayectoria lingüística: Monolingüe $P \rightarrow$ bilingüe $P-E$} \\
\hline $\begin{array}{l}\text { Socialización } \\
\text { primaria }\end{array}$ & $\begin{array}{l}\text { Socialización } \\
\text { secundaria I }\end{array}$ & $\begin{array}{l}\text { Socialización } \\
\text { secundaria II }\end{array}$ & $\begin{array}{l}\text { Vida adulta } \\
\text { inicial }\end{array}$ & Vida adulta media \\
\hline $\begin{array}{l}\text { Infancia 0-6 } \\
\text { Casa y } \\
\text { comunidad }\end{array}$ & $\begin{array}{l}\text { 6-16 } \\
\text { Escuela primaria y } \\
\text { secundaria }\end{array}$ & $\begin{array}{l}17-18 \\
\text { Escuela } \\
\text { preparatoria }\end{array}$ & $\begin{array}{l}19-23 \\
\text { Estudios } \\
\text { profesionales }\end{array}$ & $\begin{array}{l}24-50+ \\
\text { Ejercicio de la profesión }\end{array}$ \\
\hline \multirow[t]{2}{*}{$\begin{array}{l}\text { Monolingüe } \\
\text { en } \\
\text { p'urhepecha }\end{array}$} & \multirow{2}{*}{$\begin{array}{l}\text { Imposición } \\
\text { del E, trauma, } \\
\text { aprendizaje } \\
\text { limitado }\end{array}$} & \multirow{2}{*}{$\begin{array}{l}\text { Imposición del } \\
\text { E, aprendizaje } \\
\text { del E L2 con } \\
\text { dificultades }\end{array}$} & $\begin{array}{l}\text { Revaloración de la } \\
\text { cultura y lengua P } \\
\text { Reforzamiento del }\end{array}$ & $\begin{array}{l}\text { Revaloración... } \\
\text { Uso creciente y } \\
\text { mejoramiento del P como } \\
\text { lengua de instrucción }\end{array}$ \\
\hline & & & P oral y escrito & $\begin{array}{l}\text { Uso del E como L2 de } \\
\text { alumnos }\end{array}$ \\
\hline
\end{tabular}

\section{Tabla 3. Del español al p'urhepecha. Trayectoria lingüística de maestras y maestros entrevistados}

Profesores de lengua materna española: $\rightarrow$ adquisición del p'urhepecha L2

Motivación: comunicación con otros alumnos, revalorización de P en la universidad, necesidad profesional en las escuelas del proyecto.

Proceso: Menos traumático, pero no ausente de tensiones.

Resultado: bilingüismo coordinado tardío, casi siempre asimétrico.

\begin{tabular}{|c|c|c|c|c|}
\hline \multicolumn{5}{|c|}{ Trayectoria lingüística: Monolingüe $\mathrm{E} \rightarrow$ bilingüe E-P } \\
\hline $\begin{array}{l}\text { Socialización } \\
\text { primaria } \\
\text { Infancia 0-6 } \\
\text { casa y } \\
\text { comunidad }\end{array}$ & $\begin{array}{l}\text { Socialización } \\
\text { secundaria } \\
6-16 \\
\text { Escuela primaria y } \\
\text { secundaria }\end{array}$ & $\begin{array}{l}\text { Socialización } \\
\text { secundaria } \\
17-18 \\
\text { Escuela } \\
\text { preparatoria }\end{array}$ & $\begin{array}{l}\text { Vida adulta } \\
\text { inicial } \\
19-23 \\
\text { Estudios } \\
\text { profesionales }\end{array}$ & $\begin{array}{l}\text { Vida adulta media } \\
24-50+ \\
\text { Ejercicio de la profesión }\end{array}$ \\
\hline $\begin{array}{l}\text { Monolingüe } \\
\text { en español }\end{array}$ & $\begin{array}{l}\text { Educación en E } \\
\text { Creciente contacto } \\
\text { con P } \\
\text { Adquisición inicial } \\
\text { del P L2 }\end{array}$ & Educación en E & $\begin{array}{l}\text { Revaloración de la } \\
\text { cultura y lengua P } \\
\text { Continúa } \\
\text { adquisición del } \\
\text { P L2 }\end{array}$ & $\begin{array}{l}\text { Revaloración... } \\
\text { Uso creciente y mejoramiento } \\
\text { del P como lengua de } \\
\text { instrucción } \\
\text { Uso del E como L2 de alumnos }\end{array}$ \\
\hline
\end{tabular}


Otros aprendieron el purhepecha recién como docentes, cuando empezaron a atender a los alumnos monolingües en la escuela. Señalan a sus alumnos como sus "maestros" que les enseñan a diario la lengua nativa, de modo que reconocen y aceptan una reciprocidad de aprendizajes, lo que no es tan frecuente en las conceptualizaciones de maestros.

Una conclusión significativa es que la socialización lingüística y el desarrollo del bilingüismo no terminan a temprana edad; se prolongan más bien hasta la edad adulta. Este hecho contradice creencias arraigadas sobre el desarrollo del bilingüismo en contextos indígenas escolares que limitan la posibilidad de adquirir un bilingüismo avanzado en la educación primaria.

\section{4 La desatención de las necesidades lingüísticas y el surgimiento de un espacio dual de aprendizaje en la escuela indígena}

Veamos de qué manera los entrevistados tipifican lo que ocurrió en su paso por la escuela primaria, cuál fue el papel de cada lengua y qué objetivos se atienden y cuáles no.

TR 1 Maestro A

Bueno, empecé a conversar [en español] con los maestros, solito, fuera del salón, porque ahí en el salón nos daba miedo, pues, y también vergüenza, porque todos mis compañeros que, que estudiamos ahí, a veces se reían de uno cuando uno empezaba hablar el español. (Maestro, 35 años)

TR 2 Maestra B

Y empezábamos a hablar en p'urhepecha, entonces nos queríamos comunicar con los demás, pues fue que aprendimos a hablar el p'urhepecha para podernos comunicar con nuestros compañeros. Pero no estaba también permitido hablar enfrente del maestro o la monja, no se nos permitía hablar el p'urhepecha, porque ella no entendía qué hablábamos; a lo mejor estábamos hablando de ella o diciendo cosas malas, o indebidas. Entonces no estaba permitido, pero lo aprendíamos en el recreo, en la calle, a la salida o cuando no estuviera la maestra presente. (Maestra, 33 años)

TR 3 Maestro C

Desde mi infancia, desde que nací, pues mis papás me hablaron en p'urhepecha, siempre, siempre me con... tuve una comunicación, tuve comunicación en p'urhepecha $\mathrm{Al}$ igual la primaria, en la secundaria, así como le estaba diciendo, 
nunca me relacionaba en español, siempre en p'urhepecha. Entonces en bachilleres ya es cuando empecé a hablar, se me complicaba. No, no digo que así al... así al momento, pues también lo hablé [...] a pesar que yo leía los, los libros, pues, pero, no podía hablarlo, hablarlo, interpretarlo es que no podía [...] a lo mejor si hablaba algunas cosas básicas [...] pero no podía darle un seguimiento, un seguimiento, en cuanto... a la lengua hablada, pues, no, no podía hablar, no podía, se me dificultaba mucho. (Maestro, 24 años)

TR 4 Maestra D

En la casa también hablábamos en p'urhepecha, toda la comunicación era en p'urhepecha, pero no, yo nunca tuve fe en ningún maestro desde el kínder hasta... hasta en la Normal, porque tuve ya un maestro que ya nos hablaba en p'urhepecha. (Maestra, 34 años)

TR 5 Maestra B

En la casa no hablábamos el p’urhepecha. Mi papá sufrió mucho por no poder hablar el español. Tenía que salir a trabajar fuera y no se podía comunicar, se le hacía difícil, entonces no nos quiso enseñar p'urhepecha para no pasar lo mismo. Y allá en la escuela, porque aprendí con los niños a hablar el p'urhepecha con los compañeros, y en la calle $[. .$.$] entonces no estaba permitido, pero lo aprendíamos en el$ recreo, en la calle, a la salida o cuando no estuviera la maestra presente. (Maestra, 33 años)

TR 6 Maestro C

Siguiera adelante en el estudio, lo malo es que nunca aprendí a desarrollar una, una segunda lengua, cuando yo andaba en el, en la primaria... Que me enseñaban en español no me sirvió de nada, de hecho, no recuerdo [...] no, nunca me quedó nada de historia, no, no me quedó nada, al igual en la secundaria. ¿Por qué? Porque nunca desarrollé una segunda lengua, nada más leía los libros por leer, en vez, sí podía comprender, pero, pero a la vez se me dificultaba mucho hablarlo. (Maestro, 24 años)

En este conjunto de testimonios tan diversos en cuanto a las situaciones lingüísticas de partida, los entrevistados llegan a una extraordinaria coincidencia al identificar una serie de problemas estructurales en las escuelas por las que transitaron.

En primer lugar, identifican la escuela indígena como un lugar donde las necesidades lingüísticas de niños y niñas, tanto hablantes nativos del p'urhepecha como del español, no fueron contempladas o atendidas satisfactoriamente en los modelos escolares de educación indígena por donde transitaron los profesores entrevistados. La 
otra lengua no se aprende como parte central del currículo supuestamente bilingüe en las aulas, sino fuera de ellas: en el recreo, en el contacto con hablantes nativos, con el maestro o la maestra fuera del aula. Quizás no debería sorprender que los alumnos cuya lengua más fuerte es el español, no aprendan las lenguas autóctonas en la escuela indígena castellanizadora tradicional, donde se subordina o excluye la lengua indígena que además no ocupa ningún lugar curricular. Sin embargo, los alumnos que parten de la lengua indígena tampoco obtienen una enseñanza satisfactoria del español, lo que confirma la crítica de larga data que les asigna pésimos resultados a los programas de "castellanización", de sumersión o transición rápida (Hamel, 1988, 2000; Hamel et al., 2004, Instituto Nacional de Evaluación Educativa [INEE], 2008). Por lo menos, este es el resultado de las actividades focalizadas al interior del aula.

En un segundo momento, los docentes coinciden en identificar un espacio escolar extra-aula como una zona significativa donde a veces sí se aprende la otra lengua, y quizás muchas otras cosas, y con más eficiencia que en el salón. Es el territorio libre e informal del recreo y de la conversación sin marco formal y sin testigos, un espacio que abre una suerte de contra-cultura de aprendizajes y prácticas, donde caben incluso las lenguas "prohibidas" o relegadas. Habría que tomar en cuenta este espacio dual en el diseño curricular, donde se pueden alcanzar objetivos a través de estrategias, roles y prácticas bien diferenciadas.

Si bien la escuela es un territorio significativo en la construcción de la subjetividad de los maestros, las alusiones no remiten a la escuela como un lugar de aprendizaje relevante, por lo menos de las lenguas, sino hasta instancias de educación secundaria y superior. La escuela primaria aparece más como un lugar de tránsito. Y se percibe una vez más la dificultad de concebir, por parte de todos los actores, un bilingüismo de enriquecimiento como objetivo y a la vez la forma normal de convivencia que debiera expresarse en las primarias. Las escuelas indígenas que cursaron estos docentes como alumnos no constituían todavía un terreno donde el bilingüismo funcionara con naturalidad, a pesar de todos los programas y discursos institucionales que afirman lo contrario.

\subsection{Cambios y quiebres en la valoración de la lengua y cultura p'urhepecha}

En la descripción de su ciclo escolar, los maestros y maestras indígenas señalan algunos puntos de inflexión en la valoración de todo lo referido al mundo p’urhepecha. La apreciación de las lenguas parece transformarse a través de los ciclos de vida y 
Rainer Enrique Hamel, Ana Carolina Hecht, Ana Elena Erape Baltazar, Helmith Betzabé Márquez Escamilla

las etapas de la socialización escolar. Entre los maestros hablantes de p’urhepecha que sufrieron la represión en la escuela primaria y secundaria, emerge una nueva valoración positiva de su lengua y cultura en la educación superior y en el ejercicio de la profesión, junto con un mayor reconocimiento de la identidad étnica en la adultez. ${ }^{10}$ Este proceso resulta muy importante porque contradice algunos estereotipos sobre las tendencias históricas de desplazamiento. Respecto de las percepciones docentes sobre cuándo emerge lo indígena en su trayectoria, podemos citar al siguiente maestro:

\section{TR 7 Maestro A}

Para mí la escuela fue, fue, este, fue, este, $\mathrm{mmm}$, un espacio... ajeno a la... a mi familia, a mi comunidad, así, hastaaa, hasta, este, ¿cómo le diré? hasta ahí, cuando tuve ya la oportunidad de, de, de, de entrar como maestro en el Curso de Inducción [a la docencia], se empezó a hablar algo de nuestra cultura [...] pero en toda mi experiencia, este, lo que es preescolar, primaria, secundaria y bachillerato, fue una escuela, este, incómodo para mí, porque no, no se tocaba nada de, de la cultura p'urhepecha, inclusive era una vergüenza, pues, para las escuelas la cultura p'urhepecha, entonces, en todas las escuelas, para mí la escuela era miedo. (Maestro, 35 años)

La educación superior en la Escuela Normal Indígena y la UPN surge como un lugar de revalorización de la lengua e identidad indígenas. Este proceso incluyó también a los maestros p’urhepechas hispanohablantes, quienes asumieron el desafío de aprender la lengua p'urhepecha. Todos los entrevistados reportaron que adquirieron competencias de escritura en la lengua indígena durante su educación superior. Esta autoevaluación contrasta con la percepción generalizada sobre las instituciones de educación superior para indígenas en México (Escuelas normales, UpN, Universidades Interculturales) de que se logra muy poco en ellas en cuanto al aprendizaje y una revitalización real de las lenguas indígenas (López, Moya \& Hamel, 2009).

TR 8 Maestra D

Yo, de hecho, yo, hablo, hablo el p'urhepecha, pero hasta en Bachilleres, hasta en la Normal fue que... empecé a escribir, tratar de escribir en p'urhepecha, tratar de escribir en p'urhepecha porque... menos en la Prepa y en la Secundaria también, pero en la Primaria no, nunca me dieron así, todo era en, en... español. (Maestra, 34 años)

10. Ver dinámicas muy parecidas entre maestras y maestros indígenas quechuas y aimaras en los Andes, observadas por Hornberger y Swinehart (2012). 


\section{Uandakurhintskua - biografías lingüísticas}

de docentes p'urhepechas

Rainer Enrique Hamel, Ana Carolina Hecht, Ana Elena

Erape Baltazar, Helmith Betzabé Márquez Escamilla

TR 9 Maestro E

Fue en la universidad donde tomé más armas para enseñar [y escribir] nuestra lengua. (Maestro, 42 años)

Muchos de los entrevistados señalaron que desarrollaron el principal uso y la consolidación de la escritura en p'urhepecha en su experiencia como docentes (ver arriba). Es decir, la autoformación en servicio ocupa un espacio significativo en la mejora de sus competencias en p'urhepecha. Además, varias maestras y maestros indican que el principal aprendizaje en su labor profesional como docentes lo obtienen de la interacción con los niños.

TR 10 Maestro F

Con el paso del tiempo fui aprendiendo de los mismos niños, fui aprendiendo $y$, este, y así es como aprendí yo, ¿no?, pues ya llevo más de dieciocho años aquí, ya ahorita ya, ya lo... lo puedo expresar [a la lengua p’urhepecha] ya de manera más este... ora sí que natural ya, sin tanto esfuerzo y entiendo todo. (Maestro, 39 años)

Lo importante es, sin embargo, que esta orientación de revalorización etnolingüística y cultural se plasme en prácticas lingüísticas y profesionales relevantes al ingresar como docentes a las escuelas y que no permanezca como ideología enunciativa sin consecuencias. El tránsito típico de la formación docente institucional al ejercicio profesional como docente se caracteriza frecuentemente por un rápido abandono de propósitos, ideales y objetivos desarrollados en la institución formativa, una adaptación a inercias escolares y un sentimiento de impotencia por el aislamiento que perciben muchos jóvenes docentes. De ahí se explica la importancia estratégica de una organización post-formativa entre docentes y, en el caso de los profesores entrevistados, la existencia de un proyecto escolar específico, colectivo, claramente identificado como alternativa a la experiencia educativa propia que conciben como traumática y poco satisfactoria.

\subsection{Los estudios de profesorado y la superación profesional: fuente de metamorfosis y construcción de una ideología magisterial}

Llama la atención que sólo una maestra de los seis entrevistados optó por convicción por la carrera docente para su desarrollo profesional; los demás tenían otras prefe-

rencias. La carrera docente fue una opción viable, pero no una primera elección para aquellos que podían y querían seguir estudiando. Esta viabilidad se debe a que las instituciones de formación docente contemplan un acceso más fácil e incluso ayu- 
das económicas que los estudiantes indígenas no encuentran en otras instituciones universitarias. Asimismo, frente a los constreñimientos económicos y familiares, los cursos de formación docente les ofrecían en sus tiempos una rápida y segura inserción en un mercado laboral protegido. Casi todos atravesaron por un proceso que partió de la resignación ("ni modo, ya me quedé") a la aceptación del desafío y el orgullo por ejercer la profesión docente ("echarle ganas", "salir adelante”). Juega un papel importante para ellos el objetivo de perfeccionar la propia docencia para darles una mejor atención a los alumnos.

\section{TR 11 Maestro C}

Los niños son $[\ldots]$ una hoja en blanco [...], tú los puedes hacer, que si puedes hacer una buena figura, un buen papiro, uno de esos papiros [...] pero si tú lo quieres nada más arrugarlo, arrugarlo, lo puedes hacer al niño. Entonces en ese momento me di cuenta de lo importante del trabajo que llevo, de mi trabajo, lo importante de mi profesión, principalmente, lo importante que es, pues, formar a los niños, porque si yo echo a perder a un niño, todo eso se le va a quedar. (Maestro, 24 años)

Para todos los entrevistados la superación profesional ocupa un espacio central; esto incluye la formación en servicio a través de los cursos que ofrece la Secretaría de Educación Pública y de los talleres del propio programa CieıB, como también los estudios de licenciatura y maestría que se realizan generalmente los viernes y los fines de semana.

TR 12 Maestro A

[Estudiar para maestro] era por necesidad, pues, y ya después, este, me, me, me gustó, pues, y me gusta, me gusta mucho trabajar [...] [Los maestros indígenas] tenemos que trabajar diferente con nuestros alumnos para que... para que aprendan mejor, pues, y que se sien... que sientan que es, la escuela es como su casa. (Maestro, 35 años)

Un aspecto significativo de estas biografías escolares de superación personal es que en todas las historias escolares aparecen los apremios económicos como la causa del abandono o interrupción de la escuela en algún período de sus vidas. Éstas incluyen limitaciones económicas ligadas a la pérdida de alguna de las figuras paterna o materna, la imposibilidad de traslado para continuar algún ciclo escolar o la falta de equipamiento personal (vestimenta, útiles) para asistir a instituciones escolares. En síntesis, en sus historias personales, la condición económica ha significado limitaciones, pero en los casos analizados se transformaron en reinvenciones y caminos alternativos para lo proyectado. Por ello, se nota cierto orgullo en el relato de sus biografías. 
La superación personal y profesional constituye al mismo tiempo una ideología magisterial ligada al proyecto histórico de desarrollo nacional, de progreso, de ascenso social y de modernización profesional ligada a los títulos y grados universitarios. Esta ideología forma parte del repertorio magisterial nacional y es ampliamente compartida por los profesores indígenas. En el caso de los docentes entrevistados, nuestro conocimiento detallado de sus desempeños pedagógicos señala que no todos ellos practican exactamente lo que predican. Pero una comparación más exhaustiva y detallada de prácticas y representaciones, la tendremos que abordar en un trabajo futuro.

\section{Reflexiones finales: Las narrativas biográficas y las perspectivas del proyecto escolar}

El análisis de las trayectorias de vida expresadas en las narrativas autobiográficas de los docentes nos da varias pistas para reflexionar y nos permite formular algunas conclusiones y líneas de futuras investigaciones. Los temas tratados por los maestros y maestras incluyen sus trayectorias educativas y profesionales y su relación con las lenguas, la caracterización de las escuelas por donde transitaron y la construcción contrastiva de su itinerario profesional ligada a su proyecto escolar colectivo actual.

Llama la atención que todas y todos los entrevistados colocaron el tema de las lenguas en el centro de su construcción biográfica. Este hecho se torna muy significativo si consideramos que, en la metodología de la entrevista autobiográfica narrativa (EAN), se formula una pregunta inicial sin intervenciones posteriores de quien entrevista hasta que la persona entrevistada cierre su relato. Nuestra pregunta inicial no hacía referencia a las lenguas; apuntaba más bien a una reflexión amplia sobre la experiencia escolar. Ahí reside la fuerza teórico-metodológica de la EAN sustentada en el interaccionismo simbólico (Glaser \& Strauss, 1967; Schatzmann \& Strauss, 1973; Schütze, 1987): elicita las categorizaciones y el establecimiento de relevancias propias construidas por los sujetos. El hecho de que todos los entrevistados hayan colocado en el centro de su biografía educativa la cuestión de las lenguas y que hicieran alusiones a escollos vividos en la escuela por la carencia de competencias lingüísticas en la segunda lengua, sea ésta el español o el p'urhepecha, nos muestra que la lengua propia, como el bilingüismo mismo, se ubican entre los "valores nucleares" ("core values", 
Smolicz, 1981, 1994) de la identidad étnica de las comunidades p’urhepechas donde viven y trabajan. La lengua propia constituye el núcleo de la cultura p'urhe. Esta conclusión confirma muchas de nuestras observaciones y estudios previos en el campo (Hamel, 1996; Hamel \& Muñoz Cruz, 1982), como la acción pedagógica misma que, en su diseño curricular, instala la lengua p'urhepecha en el centro de las materias y competencias escolares (Hamel, 2010).

Entre las colocaciones de relevancias destaca que, en la concepción de los maestros, la socialización lingüística no se produce sólo en la niñez; la adolescencia y la adultez también constituyen etapas muy significativas para la socialización lingüística y cultural. Esto se debe en parte al hecho de que la escuela primaria, principal etapa institucional encargada de desarrollar un bilingüismo coordinado entre los alumnos, no cumple con esta función, como hemos visto en las entrevistas y también en otros estudios (Tiburcio \& Jiménez, 2016). El aprendizaje de la otra lengua se da fuera del aula o en una etapa mucho más tardía, en la preparatoria (bachillerato) o incluso en la Escuela Normal o universidad. Llama la atención que ambos grupos de maestros, tanto los que parten del p'urhepecha como los que parten del español como lengua materna, perciben este conflicto, el paso traumático por la escuela y las dificultades para la adquisición de la otra lengua, a pesar de que ambas no gozan del mismo estatus y se encuentran en una relación asimétrica de diglosia. Además, sólo la escuela primaria tiene un programa bilingüe en México, mientras que las dos etapas de educación posterior, de secundaria y preparatoria, se desenvuelven en escuelas monolingües en español.

Los testimonios de los docentes nos enseñan que la realidad educativa, sociocultural y lingüística es más compleja de lo que permiten sospechar los programas escolares formales. No solamente nos revelan lo que no se cumple en los programas, como lo es el desarrollo de un bilingüismo funcional, coordinado y enriquecedor, a pesar de que éste constituya un objetivo central; nos abren además una ventana para asomarnos a un conjunto de procesos y acontecimientos que ocurren de manera casi clandestina y a contrapelo de las tendencias sociolingüísticas dominantes (Rockwell, 2005). Estos hechos, como el aprendizaje del español fuera del aula o, más sorprendente, la adquisición del p'urhepecha por parte de los hispanohablantes en las escuelas, en la interacción con compañeros, en el recreo, nos muestran espacios soterrados de resistencia y vitalidad etnolingüística que operan a veces con asombrosa eficacia. Es un hecho reconocido en otros estudios que la adquisición de la lengua subordinada, muchas veces estigmatizada y excluida, acontece a pesar de todos los factores que 
Rainer Enrique Hamel, Ana Carolina Hecht, Ana Elena Erape Baltazar, Helmith Betzabé Márquez Escamilla

operan en su contra. Así, pudimos observar en proyectos de investigación anteriores, en zonas y escuelas indígenas donde existe un proceso avanzado de desplazamiento lingüístico y las clases se desarrollan casi exclusivamente en español, que los alumnos cuya lengua más fuerte ya es el español, mejoran su dominio de la lengua indígena de manera generalizada y conforme van avanzando a través de los grados escolares, a contrapelo de las tendencias sociolingüísticas hegemónicas (e.g. Hamel, 2009). Procesos similares se reportan en contexto de educación bilingüe de inmigrantes en los Estados Unidos y en otros países (García, 2008; Unamuno, 2005).

Ahora bien, en el caso de la región p’urhepecha, la adquisición de la lengua indígena ocurre, aunque en forma tardía e incompleta, porque preserva una vitalidad y funcionalidad lo suficientemente fuerte para constituir un polo de atracción para los maestros hispanohablantes. En caso de que su presencia social y su uso cotidiano extendido se debiliten en un futuro, es probable que la lengua indígena pierda su atractivo y que los estudiantes en formación pedagógica, como también los docentes en servicio, ya no inviertan tiempo y esfuerzo en su aprendizaje. Esto ocurre en una gran parte de las regiones indígenas en México, donde el requisito otrora indispensable de ser bilingüe para ingresar a la carrera docente indígena se diluye cada vez más.

La experiencia de una socialización etnolingüística y el desarrollo de un bilingüismo que no se consolida a temprana edad, sino que se prolonga hasta la edad adulta, se puede vincular con los resultados recientes de los estudios sobre socialización en general y socialización lingüística en particular, que abarcan cada vez más el ciclo completo de vida, ya que cada momento biográfico enfrenta y entrena a los sujetos para nuevos desafíos (Baquedano-López \& Kattan, 2007; Hecht, 2010, 2015; Luykx, 2005; Saville-Troike, 2005). Resultados como estos tienen implicaciones importantes para la futura planeación de la educación intercultural bilingüe que deberá incluir necesariamente la educación secundaria, media superior y superior.

En sus testimonios los maestros perfilan la construcción de un bilingüismo diferenciado y dinámico. Basándose en su experiencia colectiva como profesorado, aceptan que los niños indígenas pueden tener como primera lengua tanto el p'urhepecha como el español. Esta apreciación introduce un matiz significativo frente a la ideología dominante simplificadora, compartida por indígenas y no indígenas, que sólo considera indígena al que habla la lengua ancestral. Los docentes entrevistados desarrollan un modelo procesual diferenciado. No abandonan la reivindicación de un bilingüismo coordinado, enriquecedor y avanzado como objetivo ideal. Lo impor- 
tante para ellos y su contexto de acción profesional es que todos los miembros de la sociedad escolar se transformen en bilingües; que emprendan el esfuerzo de aprender la otra lengua y que sepan usar las dos lenguas en sus contextos apropiados. Sin embargo, aceptan que esta meta se alcance de un modo diversificado; admiten que no todos parten de las mismas condiciones iniciales y que el proceso no necesariamente llega a alcanzar la meta ideal. Persisten tanto la apreciación del difícil camino como también la certeza basada en la experiencia biográfica propia de que es posible construir un bilingüismo individual y colectivo, enriquecedor y estable, en medio de tanto conflicto y adversidad estructural.

Observamos que todas y todos los docentes entrevistados, incluso los de ingreso más reciente, construyen su biografía como acto de reconstrucción narrativa, de "apilamiento de experiencias" (Schütze, 1983), e instituyen sus relevancias biográficas retroactivas en clara referencia a su proyecto escolar actual. Una serie de componentes de estructuración de sus relatos biográficos, que no podemos analizar en detalle en este texto, indican claramente que los entrevistados elaboran su biografía educativa personal focalizando el proyecto escolar actual como referente central. Es decir, la experiencia profesional colectiva de su proyecto educativo, que intenta ofrecer una educación alternativa a sus alumnos, interviene como polo contrastante para estructurar y (re)valorar su propia biografía escolar y lingüística como deficiente y traumática, a partir de modelos actuales que para los maestros y maestras no solamente existen como referentes académicos abstractos, sino como una práctica cotidiana en construcción permanente, donde la experiencia personal juega un papel importante.

Esta construcción también nos da pistas para entender mejor la elaboración de narrativas biográficas en general que nunca constituyen cronologías simples, sino (re) estructuraciones y (re)valoraciones retrospectivas de etapas pasadas, a partir del posicionamiento y el lugar de enunciación actual, aquí y ahora. El corpus de entrevistas nos muestra la relación dinámica, compleja y de reciprocidades múltiples entre distintas etapas y experiencias de la vida. No sólo fue la experiencia escolar propia la que impulsó y justificó la búsqueda de un ejercicio profesional y pedagógico alternativo. La reflexión sobre la práctica actual, con sus descubrimientos casi diarios como parte de un monitoreo colaborativo, también permite reinterpretar y valorar con mayor capacidad contrastante la propia trayectoria escolar.

Como hemos visto, la conciencia de laborar y ser parte de un proyecto escolar propio constituye, sin duda, un momento unificador y orientador en las experiencias 
biográficas individuales y divergentes; establece, posiblemente, una diferencia con muchos otros docentes que no participan en un proyecto escolar propio y no perciben su práctica profesional como destacada o particularmente pertinente. Muchos maestros y maestras indígenas pasaron por procesos educativos muy similares, igualmente traumáticos y con resultados deficientes. A falta de una alternativa profesional palpable, realista y colectiva, llegan a conclusiones opuestas a las del equipo de T’arhexperakua. Frente al mismo deseo de evitarles experiencias tan dolorosas a sus alumnos y a sus propios hijos, sucumben a la ideología dominante y prefieren abandonar la lengua y cultura indígenas, tanto en su desempeño profesional como en la socialización de sus descendientes. Castellanizan y enseñan exclusivamente en español, aun cuando esta práctica, comprobada como inapropiada, lleve a resultados poco satisfactorios y reproduzca de algún modo las propias experiencias traumáticas en sus alumnos. Como sabemos, tanto el abandono de la transmisión intergeneracional de la lengua indígena, como también la práctica escolar castellanizadora, constituyen factores centrales en el desplazamiento y la desaparición de las lenguas indígenas (Hamel, 1996; Hamel et al., 2004; López, 2009a).

En cambio, los profesores de San Isidro y Uringuitiro llegaron a la conclusión contrapuesta al asumir el reto de construir una educación adecuada, intercultural y bilingüe, a pesar de no contar con ningún modelo establecido y exitoso en México. Justamente por su experiencia escolar tan negativa, los maestros y maestras del proyecto escolar formulan en sus testimonios una reivindicación de su función docente como una revancha, una suerte de resarcimiento o reparación; una segunda posibilidad que se les presenta para entablar otra relación con la escuela, y para revertir su experiencia pasada de padecimientos.

Como consecuencia de su metamorfosis, deciden mejorar su dominio oral y escrito de la lengua originaria de su pueblo, considerándola un instrumento poderoso y pertinente para la educación indígena. Se identifican positivamente con sus alumnos como miembros del mismo pueblo p'urhepecha y optan por darles una educación de mejor calidad, lo que consiste en el desarrollo sistemático de una práctica docente y de un currículo intercultural bilingüe, que coloca en el centro del quehacer escolar a su propia lengua y los conocimientos vigentes en su cultura, cuyo valor reconocieron y asumieron recién en su formación docente y el ejercicio de su profesión. Construyen de esta manera una fuerte identidad colectiva que lleva al empoderamiento de ellos mismos, de sus alumnos, de las comunidades y de un proyecto escolar que reconocen como propio. 
Rainer Enrique Hamel, Ana Carolina Hecht, Ana Elena Erape Baltazar, Helmith Betzabé Márquez Escamilla

\section{TR 13 Maestro F}

Yo les digo a mis compañeros, bueno, el compromiso es mayor de nosotros... si, si este... porque si nosotros siendo parte, no les damos... una buena educación a nuestros niños, que son nuestros hermanos, vean que son nuestros hermanos, la raza, no creo que venga otra gente, les digo (...) Nosotros somos, nosotros somos los principales protagonistas de que se dé una buena educación con nuestros hermanos de raza" (Maestro, 39 años)

\section{Lista de referencias}

Alonso, G., Alonso, P., Cano, E., \& Cortés, S. (2004). La incomprensión de la lectura en español. Proyecto: La lecto-escritura de la lengua p'urhepecha en el ámbito escolar (Tesis de licenciatura inédita). Universidad Pedagógica Nacional-Uruapan, Michoacán, San Isidro Uringuitiro, México.

Appel, M. (2005). La entrevista autobiográfica narrativa: Fundamentos teóricos y la praxis del análisis mostrada a partir del estudio de caso sobre el cambio cultural de los Otomíes en México. Forum Qualitative Sozialforschung/Forum: Qualitative Social Research, 6(2). Recuperado de http://www.qualitative-research.net/fqs-texte/2-05/05-2-16-s.htm

Baquedano López, P., \& Kattan, S. (2007). Growing up in a multilingual community: Insights from language socialization. En P. Auer \& L. Wei (Eds.), Handbook of multilingualism and multilingual communication (pp. 69-10o). Berlín y Nueva York: Mouton de Gruyter.

Dietz, G. (2012). Un esfuerzo colectivo con logros y con retos. Reseña del Proyecto T’arhexpekua-Creciendo juntos. Revista Mexicana de Investigación Educativa, $17(54), 961-964$.

García, O. (2008). Bilingual education in the 21st century: A global perspective. Malden, Massachusetts: Wiley-Blackwell.

Glaser, B. G., \& Strauss, A. L. (1967). The discovery of grounded theory. Strategies for qualitative research. Londres: Weidenfeld \& Nicolson.

Guerra, M. I. (2009). Trayectorias formativas y laborales de jóvenes de sectores populares. Un abordaje biográfico (Colección de la Educación Superior). México: Asociación Nacional de Universidades e Instituciones de Educación Superior.

Hamel, R. E. (1988). La política del lenguaje y el conflicto interétnico: Problemas de 
investigación sociolingüística. En E. P. Orlandi (Ed.), Política lingüística en América Latina (pp. 41-73). Campinas, São Paulo: Pontes.

Hamel, R. E. (1996). Conflictos entre lenguas, discursos y culturas en el México indígena: ¿la apropiación de lo ajeno y la enajenación de lo propio? En U. Klesing-Rempel (Ed.), Lo propio y lo ajeno. Interculturalidad en sociedades multiculturales (pp. 149-189). México: Plaza \& Valdés.

Hamel, R. E. (2000). Políticas del lenguaje y estrategias culturales en la educación indígena. En Instituto Estatal de Educación Pública (Ed.), Inclusión y diversidad. Discusiones recientes sobre la educación indígena en México (pp. 130-167). México: Editor.

Hamel, R. E. (2009). La noción de calidad desde las variables de equidad, diversidad y participación en la educación bilingüe intercultural. Revista Guatemalteca de Educación, 1(1), 177-230.

Hamel, R. E. (2010). Hacia la construcción de un proyecto escolar de еiв. La experiencia p'urhepecha: investigación y acción colaborativa entre escuelas e investigadores. En VIII Congreso Latinoamericano de Educación Intercultural Bilingüe (pp. 113-135). Buenos Aires: Ministerio de Educación/Fondo de las Naciones Unidas para la Infancia.

Hamel, R. E. (2016). Bilingual education for indigenous peoples in Mexico. En O. García, A. Lin \& S. May (Eds.), Bilingual and Multilingual Education, Encyclopedia of Language and Education. doi:10.1007/978-3-319-02324-3_30-2

Hamel, R. E., Brumm, M., Carrillo Avelar, A., Loncon, E., Nieto, R., \& Silva Castellón, E. (2004). ¿Qué hacemos con la castilla? La enseñanza del español como segunda lengua en un currículo intercultural bilingüe de educación indígena. Revista Mexicana de Investigación Educativa, 9(20), 83-107.

Hamel, R. E., \& Muñoz Cruz, H. (1982). Conflit de diglossie et conscience linguistique dans des communautés indigènes bilingues au Mexique. En N. Dittmar \& B. Schlieben-Lange, Die Soziolinguistik in den romanischsprachigen Ländern. La sociolinguistique dans les pays de langue romane (pp. 249-270). Tübingen: Narr.

Hamel, R. E., \& Muñoz Cruz, H. (1988). Desplazamiento y resistencia de la lengua otomí: el conflicto lingüístico en las prácticas discursivas y la reflexividad. En R. E. Hamel, Y. Lastra \& H. Muñoz Cruz (Eds.), Sociolingüística latinoamericana. X Congreso Mundial de Sociología, México 1982 (pp. 101-146). México: Universidad Nacional Autónoma de México. 
Hecht, A. C. (2010). Todavía no se hallaron hablar en idioma. Procesos de socialización lingüística de los niños en el barrio toba de Derqui, Argentina. Múnich: Lincom Europa Academic Publications.

Hecht, A. C. (2015). Trayectorias escolares de maestros toba/qom del Chaco. Cuadernos del Instituto Nacional de Antropología y Pensamiento Latinoamericano, $24(2), 1-12$.

Hornberger, N. H., \& Swinehart, K. F. (2012). Not just situaciones de la vida: Professionalization and indigenous language revitalization in the Andes. Multilingual Research Journal, 6(1), 35-49.

Instituto Nacional de Evaluación Educativa. (2008). La educación para poblaciones en contextos vulnerables. México: Autor.

Kohler, C. (1993). Narrative analysis. Londres: Sage.

Lafont, R. (1979). Productivité culturelle et domination linguistique. Lengas, 6, 1-22.

Lafont, R. (1980). La spectacularisation de l'occitanophonie dans les enquêtes sociolinguistiques : la fonction du 'retour'. Lengas, 7, 7-78.

López, L. E. (Ed.) (2009a). Interculturalidad, educación y ciudadanía. La Paz: FUnPRoeib Andes \& Plural.

López, L. E. (2009b). Reaching the unreached: indigenous intercultural bilingual education in Latin America. Education for All Global Monitoring Report 2010. Washington.

López, L. E., Moya, R., \& Hamel, R. E. (2009). Pueblos indígenas y educación superior en América Latina y el Caribe. En L. E. López (Ed.), Interculturalidad, educación y ciudadanía. Perspectivas latinoamericanas (pp. 221-289). La Paz: FUnPRoeib Andes \& Plural.

Luykx, A. (2005). Children as socializing agents: family language policy in situations of language shift. En J. M. Cohen, T. Kara, K. Rolstad \& J. MacSwan (Eds.), Proceedings of the 4 th International Symposium on Bilingualism (pp. 1407-1414). Massachusetts: Cascadilla Press.

Manessy, G., \& Wald, P. (1984). Le français en Afrique noire. Tel qu'on le parle, tel qu'on le dit. París: L'Harmattan-IDERIC.

Muñoz Cruz, H. (1983). ¿Asimilación o igualdad lingüística en el Valle del Mezquital? Nueva Antropología, 22, 25-64.

Muñoz Cruz, H. (2006). Niveles y tópicos de reflexividad sociolingüística a través de autobiografías. Signos Lingüísticos, 3, 69-91.

Muñoz Cruz, H. (2009). Reflexividad sociolingüística de hablantes de lenguas indígenas: 
Rainer Enrique Hamel, Ana Carolina Hecht, Ana Elena Erape Baltazar, Helmith Betzabé Márquez Escamilla

concepciones y cambio sociocultural (Biblioteca Signos). México: Universidad Autónoma Metropolitana.

Riemann, G., \& Schütze, F. (1991). "Trajectory” as a basic theoretical concept for analyzing suffering and disorderly social processes. En D. R. Maines (Ed.), Social organization and social process. Essays in honour of Anselm Strauss (pp. 333-357). Nueva York: Aldine de Gruyter.

Rockwell, E. (2005). La apropiación, un proceso entre muchos que ocurren en ámbitos escolares. Memoria, conocimiento y utopía. Anuario de la Sociedad Mexicana de Historia de la Educación, 1. 28-38.

Saville-Troike, M. (2005). Etnografía de la comunicación. Buenos Aires: Prometeo.

Schatzman, L., \& Strauss A., L. (1973). Field research. Strategies for natural sociology. Englewood Cliffs, N. J: Prentice-Hall.

Schütze, F. (1983). Biographieforschung und narratives Interview. Neue Praxis, 13(3), 283-293.

Schütze, F. (1987). Symbolischer Interaktionismus. En U. Ammon, N. Dittmar \& K. J. Mattheier (Eds.), Sociolinguistics/Soziolinguistik (pp. 520-553). Berlín y Nueva York: Walter de Gruyter.

Schütze, F. (1992a). Pressure and guilt: War experiences of a young German soldier and their biographical implications (Part 1). International Sociology, 7(2), 187-208.

Schütze, F. (1992b). Pressure and guilt: War experiences of a young German soldier and their biographical implications (Part 2). International Sociology, 7(3), 347-367.

Serrano Castañeda, J. A., \& Ramos Morales, J. M. (2014). Boceto del espacio biográfico-educativo en México. Revista Mexicana de Investigación Educativa, 19(62), $831-858$.

Sierra, M. T. (1992). Discurso, cultura y poder. El ejercicio de la autoridad en los pueblos hñähñus del Valle del Mezquital. México: Centro de Investigaciones y Estudios Superiores en Antropología Social/Gobierno del Estado de Hidalgo.

Smolicz, J. (1981). Core values and cultural identity. Ethnic and racial studies, 4(1), 7590.

Smolicz, J. (1994). Australia's language policies and minority rights: a core value perspective. En T. Skutnabb-Kangas \& R. Phillipson (Eds.), Linguistic human rights (pp. 235-252). Berlín y Nueva York: Mouton de Gruyter.

Souza de, E. C., Serrano, J. A., \& Ramos, J. M. (2014). Autobiografía y educación. Tradiciones, diálogos y metodologías. Revista Mexicana de Investigación Educativa, 19(62), 683-694. 
Tiburcio, C., \& Jiménez, Y. (2016). Un caso atípico en la enseñanza de lenguas indígenas: el tutunakú de Coahuitlán, Veracruz. Revista Interamericana de Educación de Adultos, 38(1), 33-47.

Unamuno, V. (2005). L'entorn sociolingüístic i la construcció dels repertoris lingüístics de l'alumnat immigrat a Catalunya. Noves SL. Revista de sociolingüística, 1, Recuperado de http://www.gencat.cat/llengua/noves/noves/hmosprimavera-estiu/unamuno1_3.htm

Woolard, K. A. (1998). Introduction: Language ideology as a field of inquiry. En B. SchieffelinK, A.Woolard \& P. V. Kroskrity (Eds.), Language ideologies. Theory and practice (Trad. M. Rodríguez) (pp. 3-47). Oxford: Oxford University Press.

Los textos de R. E. Hamel son accesibles en www.hamel.com.mx 